\title{
Enjeux et amélioration de la réduction de l'acidité dans les fruits mûrs du palmier à huile, Elaeis guineensis Jacq. (synthèse bibliographique)
}

\author{
Hubert Domonhédo ${ }^{(1,2,3)}$, David Cros ${ }^{(4)}$, Léifi Nodichao ${ }^{(1)}$, Norbert Billotte ${ }^{(4)}$, \\ Corneille Ahanhanzo ${ }^{(3)}$
}

\author{
(1) Centre de Recherches Agricoles Plantes Pérennes (CRAPP). Institut National des Recherches Agricoles du Bénin. \\ BP 1 Pobè (Bénin). E-mail : hubertdomonhedo@yahoo.fr \\ (2) Université d'Abomey-Calavi. Faculté des Sciences Agronomiques. 01 BP 526 Cotonou (Bénin). \\ (3) Université d'Abomey-Calavi. Faculté des Sciences et Techniques (FAST). Département de Génétique et des \\ Biotechnologies. 01 BP 526 Cotonou (Bénin). \\ (4) CIRAD. UMR AGAP (Unité Mixte de Recherches Amélioration Génétique et Adaptation des Plantes méditerranéennes et \\ tropicales). FR-34398 Montpellier (France).
}

Reçu le 13 mars 2017, accepté le 29 septembre 2017, mis en ligne le 6 octobre 2017.

Cet article est distribué suivant les termes et les conditions de la licence CC-BY (http://creativecommons.org/licenses/by/4.0/ deed.fr)

Introduction. L'acidification de l'huile de palme détermine la qualité et la stabilité de cette importante denrée alimentaire. Cette synthèse analyse les causes de l'acidification de l'huile et son impact sur la qualité et la stabilité de l'huile. Les enjeux liés à la réduction de l'acidification de l'huile et les approches utilisées sont aussi analysés, en particulier la réduction par l'amélioration génétique.

Littérature. L'acidification est principalement due à l'action de la lipase endogène du mésocarpe, mais peut aussi être causée par des lipases microbiennes ou une hydrolyse autocatalytique. Plusieurs facteurs, notamment le matériel végétal, les conditions de récolte et de traitement post-récolte des régimes, d'extraction et de conservation de l'huile impactent de manière significative l'acidification de l'huile. L'acidification réduit la qualité et la valeur marchande de l'huile et engendre une baisse de productivité. Des fonds génétiques à faible acidité ont été identifiés. La variabilité de ce caractère rend possible la sélection variétale. Un gène impliqué dans l'acidification de l'huile est identifié, mais l'action d'autres gènes ou facteurs génétiques est soupçonnée.

Conclusions. Ces recherches ont permis la récente commercialisation des premiers palmiers avec une huile faiblement acide. Ceci améliorera la qualité de l'huile tout en augmentant le rendement et en facilitant la gestion des opérations de récolte et de post-récolte, en particulier pour les petits producteurs. Il est nécessaire de continuer la recherche de tous les facteurs génétiques impliqués au niveau du genre Elaeis. La validation des ressources génomiques permettrait la sélection assistée par marqueurs de variétés à faible acidité de l'huile.

Mots-clés. Huile de palme, qualité, amélioration génétique, acidité, lipase, palmier oléifère.

Reduction of acidity in mature oil palm (Elaeis guineensis Jacq.) fruits: stakes and oil quality improvement. A review Introduction. Palm oil acidification determines the quality and stability of this important foodstuff. This review article analyzes the causes of acidification, and its effects on the quality and stability of palm oil. The stakes involved in the reduction of acidity in fruits and the approaches used are also analyzed, especially reduction via genetic improvement.

Literature. Acidification is mainly due to the action of an endogenous lipase of the fruit's mesocarp, but it can also be caused by microbial lipase or autocatalytic hydrolysis. Many factors, particularly plant material, conditions of harvest and postharvest bunch treatment, and conditions of oil extraction and storage have an appreciable impact on acidification. Acidification reduces the quality and market value of palm oil and causes important oil losses due to the removal of free fatty acids in the mill. The genetic background of individuals of low oil acidity has been identified. The variability of this trait makes varietal selection possible. One gene implicated in palm oil acidification has been identified, but other genes or genetic factors may also be involved. 
Conclusions. Such research has led to the recent commercialization of the first oil palms with low acidity. This will improve oil quality, increase yield and facilitate the management of harvest and post-harvest operations, especially for smallholder farmers. There is a need to identify all the genetic factors responsible for the degradation of triglycerides in the Elaeis genus. The validation of genomic tools would allow the marker-assisted selection of oil palm varieties with low acidity.

Keywords. Palm oils, quality, breeding, acidity, lipases, oil palms.

\section{INTRODUCTION}

Le palmier à huile (Elaeis guineensis Jacq.) est la plante oléagineuse cultivée qui fournit le plus gros rendement, avec en moyenne $3,8 \mathrm{t} \cdot \mathrm{ha}^{-1} \cdot \mathrm{an}^{-1}$ d'huile de palme (Rival \& Levang, 2014 ; Corley \& Tinker, 2016). Ce rendement peut atteindre 7 à $9 \mathrm{t} \cdot \mathrm{ha}^{-1} \cdot \mathrm{an}^{-1}$ dans les conditions agropédoclimatiques les plus favorables (Durand-Gasselin et al., 2010 ; Durand-Gasselin et al., 2011). Le palmier à huile produit deux types d'huile : l'huile de palme et l'huile de palmiste. La production a fortement progressé ces dernières décennies. En 2016, elle est estimée à environ 60 millions de tonnes d'huile de palme et 7 millions de tonnes d'huile de palmiste (USDA, 2017). La seule huile de palme représente plus du tiers de la production totale de corps gras végétaux. Cette performance résulte essentiellement d'un rendement à l'hectare très élevé.

Les travaux d'amélioration variétale ont essentiellement porté sur l'augmentation du rendement en huile de palme (Demol et al., 2002; Corley \& Tinker, 2016) et ont abouti à un progrès génétique estimé à $1 \%$ par an (Durand-Gasselin et al., 2010). De plus en plus, l'amélioration du rendement en huile par unité de main-d'œuvre, indispensable aux activités principalement manuelles (entretien et récolte des plantations, extraction d'huile, etc.) et celle de la qualité alimentaire de l'huile de palme (composition en acide gras, faible acidité, etc.) prennent une part croissante dans les objectifs de sélection, suite aux demandes des planteurs et des sociétés consommatrices plus exigeantes. L'huile de palme est constituée à plus de $95 \%$ en masse de triglycérides ou triacylglycérols (TAG) provenant de l'estérification des trois fonctions alcool du glycérol par trois acides gras. La plupart des acides gras sont mis en réserve sous forme de TAG chez le palmier à huile (Sambanthamurthi et al., 2000a). Les constituants mineurs représentent moins de $5 \%$ en masse de la composition de l'huile de palme. Il s'agit de mono- et diacylglycérols, de phosphatides, ester de stérols, pigments, tocophérols, traces de métaux, etc. Il peut aussi exister dans l'huile des acides gras libres (AGL) qui proviennent de l'hydrolyse de ces triglycérides. L'hydrolyse est la réaction inverse de l'estérification, la décomposition des TAG par fixation de molécules d'eau. Les liaisons ester de la molécule de TAG sont rompues et les ions $\mathrm{H}^{+}$et $\mathrm{OH}^{-}$se fixent pour former, à l'issue d'une première phase d'hydrolyse partielle, des diglycérides et des AGL puis à la fin de la réaction, du glycérol et des AGL. Cette réaction peut être catalysée par des lipases présentes dans le mésocarpe des fruits et chez des micro-organismes associés aux fruits, et être autocatalysée en présence d'eau.

La teneur en AGL détermine l'acidité de l'huile, le principal indicateur de qualité alimentaire et de stabilité, définissant l'acceptabilité et la valeur marchande de l'huile. Au-delà de $5 \%$ d'acides gras libres, l'huile de palme, sans raffinage approprié, n'est pas recommandée pour la consommation (Codex Alimentarius, 2015). Plusieurs facteurs aussi bien intrinsèques qu'exogènes au matériel végétal peuvent affecter l'importance de cette acidification, en particulier le niveau d'activité de la lipase endogène du mésocarpe du fruit et des facteurs liés aux conditions de récolte des régimes et d'extraction de l'huile (retards à la récolte, blessure des fruits, conditions favorables au développement de micro-organismes, etc.). Le facteur prépondérant est cependant la lipase endogène du mésocarpe (Sambanthamurthi et al., 1995; Ngando Ebongue, 2009 ; Morcillo et al., 2013 ; Ngando Ebongue et al., 2013). L'acidification de l'huile de palme sous l'effet de la lipase endogène du mésocarpe des fruits a donc une importance majeure pour la filière. L'intérêt économique de l'acidité de l'huile de palme a fait de la lipase endogène du mésocarpe l'enzyme la plus étudiée chez le palmier à huile (Sambanthamurthi et al., 2000a). Ce sujet a connu de récents développements notamment avec l'identification de fonds génétiques comportant des individus à faible acidité de l'huile (Ngando Ebongue et al., 2008 ; Wong et al., 2015), puis la mise en évidence et la caractérisation d'un gène déterminant le niveau d'acidité (Morcillo et al., 2013 ; Nurniwalis et al., 2015 ; Wong et al., 2015 ; LikengLi-Ngue et al., 2016). Afin de faire évoluer les variétés commerciales actuelles, essentiellement E. guineensis à forte acidité (Ngando Ebongue et al., 2008 ; Cadena et al., 2013 ; Morcillo et al., 2013), vers des variétés à faible acidité, il est important de faire le point sur les connaissances actuelles et discuter des recherches qui restent à faire. Cette synthèse analyse les causes de l'acidification de l'huile et son impact sur la qualité et la stabilité de l'huile. Les enjeux liés à la réduction de l'acidification de l'huile et les approches utilisées sont aussi analysés, en particulier la réduction par l'amélioration génétique. 


\section{CAUSES DE L'ACIDIFICATION DE L'HUILE DE PALME}

Chez le palmier à huile, une lipase endogène exprimée dans le mésocarpe du fruit provoque une dégradation rapide des triglycérides en libérant des acides gras lors de l'abscission ou de la blessure du fruit mûr, ce qui occasionne l'acidification de l'huile de palme (Desassis, 1957 ; Abigor et al., 1985 ; Henderson et al., 1991 ; Ngando Ebongue et al., 2006).

Les triacylglycérol-lipases sont des enzymes capables d'hydrolyser les triglycérides contenant des acides gras à longues chaines (16-18 atomes de carbone) (Egloff et al., 1995). Elles appartiennent à la superfamille des hydrolases à repliement alpha/ beta qui contiennent une triade catalytique ser-asp/ glu-his. Il existe plusieurs familles de triacylglycérol lipases dont la séquence diverge fortement mais dont la structure tridimensionnelle est similaire et seul un motif est plus ou moins conservé autour de la sérine catalytique (motif PROSITE PS0120). Néanmoins, ce motif peut apparaitre dans des hydrolases n'agissant pas sur les triglycérides et être absent de la séquence de triacylglycérol lipases avérées. On ne peut donc pas prédire une activité lipase en se basant seulement sur la séquence d'une protéine, mais la mesure d'une activité lipase en utilisant un substrat naturel (triacylglycérol à longue chaine) est indispensable. En l'absence d'eau libre, les lipases peuvent catalyser la réaction inverse (estérification d'acide gras sur un alcool) (Hills et al., 1990 ; Fickers et al., 2008), cette propriété est utilisée pour le bio-façonnement des corps gras. Chez les plantes, les lipases les plus étudiées sont impliquées dans la mobilisation des huiles de réserve des plantes oléagineuses au cours de la croissance post-germinative (Eastmond, 2006 ; Kelly et al., 2016). D'autres triacylglycérol lipases ont été détectées en abondance dans d'autres tissus de plantes (latex, mésocarpe de fruit de palme, etc.) et leur fonction physiologique reste inconnue.

L'action de lipases de micro-organismes dans l'acidification de l'huile de palme a été mise en évidence à travers différentes études (Hiol et al., 2000 ; Abbas et al., 2002). Ces micro-organismes commencent à attaquer les fruits matures de palmier à huile détachés ou blessés et causent une augmentation de la teneur en AGL (Corley \& Tinker, 2016). Leur prolifération est due souvent à de mauvaises pratiques créant des conditions qui leur sont favorables (détérioration des fruits, forte température ou humidité, délai entre récolte et stérilisation, entassement des régimes avant stérilisation, etc.).

Par ailleurs, en présence d'eau, les AGL déjà présents en petite quantité agissent comme catalyseurs dans la réaction entre les triglycérides et l'eau, générant d'autres AGL (Ngando Ebongue, 2009). C'est un processus qui se développe essentiellement au cours du stockage et même en l'absence de toute activité enzymatique, dans l'huile saturée en eau (0,4\% d'humidité) (Loncin \& Jacobsberg, 1963 ; Corley \& Tinker, 2016). Selon le Codex Alimentarius (2015), la teneur en eau de l'huile doit être inférieure à $0,1 \%$. En dessous de ce taux, l'hydrolyse auto-catalytique et toute action hydrolytique d'éventuelles lipases microbiennes sont négligeables (de Graaf, 1976 ; Berger, 1983 ; Chong, 2000). À la suite de l'extraction, un bon séchage de l'huile avant stockage est alors recommandé (Corley \& Tinker, 2016). La lipase endogène reste le facteur le plus important dans l'acidification de l'huile de palme.

\section{EFFETS DES CONDITIONS DE RÉCOLTE ET DE POST-RÉCOLTE SUR L'ACIDIFICATION}

Pour limiter l'acidification de l'huile de palme, il faut maintenir une fréquence élevée de récolte des régimes mûrs, tous les 7 à 10 jours (Ngando Ebongue et al., 2008 ; Morcillo et al., 2013) et les stériliser rapidement afin d'inactiver la lipase endogène et de neutraliser les micro-organismes. La fréquence des récoltes est fonction des moyens (superficies plantées, disponibilité en main-d'œuvre, etc.), qui ne permettent pas toujours de respecter la fréquence requise, en particulier chez les petits producteurs. Dans ce cas, une importante partie de la récolte est constituée de fruits détachés qui contribuent à accroitre l'acidification de l'huile. Pour apprécier l'impact d'une récolte tardive sur l'acidification de l'huile, Ngando Ebongue (2009) a déterminé l'acidité de l'huile issue des fruits tombés par abscission à différents jours après la date normale de récolte et a observé que l'acidité reste inférieure à $3 \%$ jusqu'au 18 jour chez des palmiers préalablement caractérisés comme à faible activité lipase, tandis qu'elle oscille entre 11 et $30 \%$ chez les palmiers à forte activité lipase. Des résultats similaires ont été trouvés par Morcillo et al. (2013). Ils ont montré que les régimes d'individus à faible acidité peuvent attendre jusqu'à une dizaine de jours avant l'extraction et donner une huile dont l'acidité est toujours en deçà de $5 \%$.

L'acidification de l'huile dépend aussi du temps écoulé après la récolte des régimes avant de les traiter et d'extraire l'huile. Pour éviter la dégradation qualitative, en particulier l'acidification de l'huile, il faut procéder rapidement à la cuisson des régimes récoltés et à l'extraction de l'huile. Ceci fait appel à d'importants moyens (transport, main-d'œuvre) et crée parfois des surcouts. Les petits transformateurs, qui vont souvent récolter ou acheter des régimes loin de leur unité de transformation, n'arrivent pas toujours à remplir ces conditions difficiles. Ces petits transformateurs utilisent 
aussi des méthodes de traitement et d'extraction de l'huile qui tendent à accentuer l'activation et l'expression de la lipase (Ngando Ebongue et al., 2011). Plusieurs auteurs (Soumanou, 1986; Orji \& Mbata, 2008 ; Ngando Ebongue et al., 2011) ont indiqué que les procédés artisanaux de transformation des régimes donnent une huile de moindre qualité, plus acide et plus oxydable comparés aux procédés semi-mécaniques ou mécaniques qui permettent d'obtenir une huile avec moins d'AGL et d'impuretés et de faible teneur en eau. Les ensembles agro-industriels peuvent donc techniquement fournir une meilleure qualité d'huile de palme que celle produite à l'échelle artisanale ou semiindustrielle. Or, les petits producteurs ou propriétés familiales qui utilisent encore des procédés artisanaux de transformation, et dont certains avec parfois des presses et autres petits équipements, constituent une composante clé de la chaine de production de l'huile de palme. La non-prise en compte de la proportion et de la qualité de l'huile produite par ceux-ci serait un risque pour la filière. Selon Vermeulen \& Goad (2006), leur part dans la production était de $30 \%$ au niveau mondial et pouvait atteindre $80 \%$ au Nigéria, le plus grand producteur en Afrique. Elle peut atteindre $90 \%$ en Afrique de l'Ouest où l'huile de palme est essentiellement produite pour les marchés locaux et régionaux (Vermeulen \& Goad, 2006). Au milieu de l'année 2016, cette même catégorie d'acteurs de la filière a produit $40 \%$ de la quantité totale d'huile de palme (RSPO, 2017). La Conférence des Nations unies sur le développement et le commerce estime qu'environ la moitié de la production d'huile de palme provient des petits planteurs, dont la contribution peut varier selon les pays (98\% en Thailande, $90 \%$ au Ghana, $40 \%$ en Indonésie et $39 \%$ en Malaisie) (CNUCED, 2016). Face à la forte demande en huiles végétales prévue pour doubler d'ici 2030, il est espéré une croissance de la proportion d'huile de palme (Murphy, 2009 ; Carlsson et al., 2011). Toutefois, la majeure partie de la production d'huile de palme des pays en développement, notamment en Afrique, n'est pas compétitive sur les marchés internationaux (Ohimain et al., 2012). Des défis se présentent ainsi à la production et à la commercialisation de l'huile de palme et sont liés à l'obtention d'un produit de qualité respectant les normes en vigueur pour un accès plus facile au marché.

Plusieurs facteurs liés aux pratiques de gestion des récoltes, au traitement des régimes et au processus d'extraction et de stockage de l'huile contribuent énormément à une mauvaise qualité et stabilité de l'huile. L'acidité est fortement influencée par la blessure des fruits lors de la récolte et du long délai de transport des régimes, ainsi que du temps de stockage des régimes avant leur traitement pour inactiver la lipase endogène. De même, le manque de soin dans le processus de préparation de l'huile afin d'éliminer ou de réduire la teneur en eau et la charge microbienne, impacte sensiblement l'acidité de l'huile. Il est important de réduire la durée entre la récolte des régimes et leur transformation ainsi que d'améliorer les conditions de traitement et de réduire la teneur en eau de l'huile pour amoindrir ou pallier l'impact de ces facteurs sur l'acidification et la qualité de l'huile de palme.

\section{EFFETS NÉGATIFS DE L'ACIDIFICATION DE L'HUILE}

L'huile comportant au-delà de $5 \%$ d'AGL est considérée comme impropre à la consommation (Codex Alimentarius, 2015). La stabilité de l'huile au cours de la conservation dépend de sa qualité initiale et des conditions de stockage. L'huile acide (AGL $>5 \%$ ), déjà de mauvaise qualité, ou contenant une humidité résiduelle élevée $(>0,1 \%)$, se dégrade plus rapidement. En effet, les AGL ont un impact négatif tout au long du processus de production de l'huile et lors de sa conservation. Une teneur élevée en AGL stimule l'acidification lors de la conservation, elle favorise une hydrolyse auto-catalytique où les AGL déjà présents interviennent comme catalyseurs dans la réaction entre les TAG et l'eau pour générer d'autres AGL (Ngando Ebongue, 2009). Par ailleurs, les AGL, une fois libérés des triglycérides, sont plus sensibles au processus de peroxydation attaquant les doubles liaisons des acides gras insaturés qui se fragmentent en générant des aldéhydes et des cétones (Ngando Ebongue et al., 2011; Ngando Ebongue et al., 2013). Cette dégradation oxydative des AGL (rancissement) modifie la qualité de l'huile qui se dégrade en dégageant une fort mauvaise odeur et prend une saveur âcre, désagréable (Sambanthamurthi et al., 2000a ; Ngando Ebongue et al., 2011 ; NgandoEbongue et al., 2013). Ceci impacte négativement la valeur marchande du produit et son acceptation. Bien qu'il soit possible d'éliminer ou de neutraliser les AGL lors du raffinage, cela entraine un surcout et une perte de rendement d'huile (Gibon et al., 2007). Par ailleurs, les molécules de diacylglycérols demeurent et ont d'importants effets indésirables sur la cristallisation et le fractionnement de l'huile (Corley \& Tinker, 2016). De même, une teneur élevée en AGL réduit le taux de blanchiment de l'huile (Parakh et al., 2000).

L'objectif du raffinage est de débarrasser l'huile de tout ce qui est indésirable tel que les AGL, impuretés, odeur, gout, couleur, etc. tout en essayant de perdre le moins possible de composantes désirables et bénéfiques (tocophérols, tocotriénols, caroténoïdes, vitamines) (Gibon et al., 2007 ; Gibon et al., 2009). L'efficience et le rendement du raffinage dépendent en grande partie 
de la teneur en AGL et d'autres paramètres de qualité de l'huile brute (Gibon et al., 2009). Deux voies sont généralement utilisées, à savoir un procédé physique et un procédé chimique, qui diffèrent essentiellement selon la technique d'élimination des AGL. Le procédé physique permet d'éliminer les AGL par une distillation conjointe à la désodorisation effectuée par injection de vapeur d'eau dans l'huile à haute température et sous vide poussé. Il est préféré au procédé chimique où la neutralisation alcaline qui permet d'éliminer les AGL par transformation en savons et séparation occasionne beaucoup de pertes d'huile dans le cas d'une acidité élevée (Gibon et al., 2009). Il serait intéressant d'analyser le degré de faisabilité d'un tel traitement (élimination des AGL) dans les procédés traditionnels afin d'améliorer la qualité de l'huile.

Les procédés de transformation utilisés par les petits producteurs ou propriétés familiales ne tiennent pas compte de ces moyens et techniques d'élimination ou de neutralisation des AGL. Concernant la voie physique d'élimination des AGL, sa faisabilité dépend des moyens et de la taille de l'unité de transformation car la mise en application de cette méthode peut requérir un certain cout d'acquisition d'équipements à adapter et la consommation d'énergies électrique ou autre. En effet, il existe déjà la pratique de chauffage de bois pour porter l'huile à haute température, mais il faudrait disposer d'équipements adaptés pour pulvériser l'eau dans l'huile chaude. La difficulté réside aussi dans la quantité d'huile à traiter et la possibilité de fournir la pression nécessaire (sous vide).

Quant à la neutralisation alcaline, les phases nécessaires comprennent l'addition d'une solution d'hydroxyde de sodium (soude), le mélange, la séparation par centrifugation, des lavages à l'eau, la séparation puis le séchage sous vide.Les transformateurs ont déjà l'habitude d'utiliser de la soude pour fabriquer du savon, mais dans ce cas, l'objectif est tout autre car selon la quantité d'huile, une proportion donnée de soude est apportée pour obtenir essentiellement du savon. Dans la perspective d'éliminer les AGL et d'obtenir de l'huile de meilleure qualité, il peut être craint d'avoir une huile alimentaire mélangée à du savon ou contenant des produits chimiques. Ici, également, l'on pourrait envisager l'utilisation d'un centrifugeur adapté à cause de la rotation à grande vitesse qui ne peut être faite manuellement.

La faisabilité de ces traitements dans les procédés traditionnels est faible car l'adaptation de certaines techniques va nécessiter la mise au point d'équipements adéquats et un investissement substantiel. Il ressort un rôle important à jouer par des équipes de recherchedéveloppement et des concepteurs afin d'adapter des outils et équipements convenables, simples et à la portée de ces acteurs de la chaine. Cependant, il faut rappeler que le but est d'optimiser les procédés traditionnels en utilisant certaines techniques pour l'élimination des AGL et non abandonner subitement les procédés traditionnel ou semi-artisanal pour l'industrialisation.

D'ores et déjà, il est important de sensibiliser ces acteurs afin qu'une attention particulière soit déjà accordée aux aspects à la base de l'acidification (indiqués dans l'introduction et le point 2) lors des différentes étapes de l'extraction artisanale pour éviter l'augmentation des AGL et obtenir une huile brute de meilleure qualité.

\section{MESURE DE L'ACIDITÉ DE L'HUILE : TESTS COLORIMÉTRIQUES VARIÉS}

\subsection{Mesure de l'acidité}

Pour quantifier le degré d'acidification de l'huile, plusieurs terminologies sont utilisées : indice d'acide, acidité, teneur en AGL, etc. L'indice d'acide représente le nombre de milligrammes d'hydroxyde de potassium nécessaires pour neutraliser les AGL présents dans $1 \mathrm{~g}$ de corps gras ; il s'exprime en $\mathrm{mg}$ de $\mathrm{KOH} \cdot \mathrm{g}^{-1}$. La teneur en AGL représente l'ensemble des AGL contenus dans l'huile. Cette teneur est souvent connue sous le nom d'acidité totale ou d'acidité libre ou d'acidité. Elle est exprimée en pourcentage de masse d'acide palmitique, l'acide gras majoritaire de l'huile de palme. La teneur en AGL acceptée selon la norme internationale doit être inférieure à $5 \%$ (Codex Alimentarius, 2015). Plus l'huile est acide, plus la valeur de ces indicateurs est élevée.

Techniques couramment utilisées dans les filières agro-industrielles. La teneur en AGL de l'huile de palme est souvent déterminée par titrage en utilisant des solvants (Haryati \& Buana, 2003), en mesurant la quantité d'hydroxyde de potassium nécessaire pour neutraliser les AGL dans $1 \mathrm{~g}$ d'huile ou de graisse. C'est notamment le cas des méthodes NF EN ISO 660 et AOCS Ca 5a-40 de détermination de la teneur en AGL selon respectivement l'Organisation internationale de normalisation et la Société américaine des chimistes des huiles et qui sont celles recommandées par le Codex Alimentarius. Il s'agit d'un titrage par une solution alcoolisée d'hydroxyde de potassium de l'échantillon d'huile dissout dans un mélange de solvants. Les solvants et l'alcool utilisés dépendent de la méthode.

Autres techniques. Il existe d'autres méthodes de titrage (Haryati \& Buana, 2003) ainsi que plusieurs équipements ou appareils pour apprécier l'acidité en routine. Le «palm oil tester » (CDR Srl, Firenze, Italie, www.palmoiltester.com), par exemple, est un appareil de mesure de différents paramètres de qualité 
de l'huile de palme, dont l'acidité. Dans ce cas, les acides gras libres réagissent avec un composé à base de chrome en affaiblissant l'intensité de sa couleur. Cette diminution de couleur, lue à $630 \mathrm{~nm}$, est proportionnelle à la concentration acide (\% d'acide palmitique) de l'échantillon.

Les méthodes de titrage ne sont pas spécifiques: elles ne peuvent différencier les acides minéraux, les AGL et autres acides organiques. Ce n'est pas un problème dans notre cas car les échantillons d'huile sont obtenus par extraction à l'hexane, ce qui exclut les acides minéraux et organiques polaires. Une alternative serait d'utiliser des méthodes permettant de quantifier directement les acides gras, comme la chromatographie en phase gazeuse, mais il reste nécessaire de séparer les acides gras libres des lipides complexes avant l'analyse. Également, des techniques basées sur l'infrarouge peuvent être employées (Haryati \& Buana, 2003). Ces méthodes sont plus couteuses, nécessitent une grande technicité et n'apportent rien de plus pour la mesure de l'acidité de l'huile que les techniques de titration.

\subsection{Mesure de l'activité lipase}

Techniques de mesure de l'activité lipase. Il est important d'utiliser un substrat naturel (triacylglycérol à longue chaine) pour mesurer l'activité lipase (Beisson et al., 2000). En effet, les substrats artificiels solubles peuvent souvent être hydrolysés par des hydrolases qui n'attaquent pas les triglycérides à longue chaine et ces hydrolases peuvent être beaucoup plus abondantes que les triacylglycérol lipases (Huang, 1993). En utilisant des triacylglycérols à longues chaines comme substrat, il est important de s'assurer qu'ils soient correctement émulsifiés pour que le substrat ne soit pas limitant.

De nombreuses techniques sont disponibles pour mesurer l'activité lipase (Beisson et al., 2000), mais le $\mathrm{pH}$-stat reste la technique de référence dès que l'activité lipase est supérieure à $0,1 \mu$ mol d'acide gras libéré par minute. Elle permet de mesurer en temps réel les cinétiques d'hydrolyse du substrat par la lipase en additionnant continuellement un réactif pour neutraliser les ions $\mathrm{OH}^{-}$ou $\mathrm{H}_{3} \mathrm{O}^{+}$afin de maintenir le $\mathrm{pH}$ stationnaire, d'où le nom « $\mathrm{pH}$-stat». Il s'agit d'un dispositif comportant un pot thermostaté, une électrode de $\mathrm{pH}$, un agitateur pour remuer constamment l'émulsion de TAG injectée dans le pot et une burette contenant le réactif titrant qui permet de maintenir le $\mathrm{pH}$ constant à la valeur de consigne lors de la réaction enzymatique. L'action de la lipase engendre la libération d'une quantité d'acides gras qui est proportionnelle à la quantité de soude versée et cette activité peut être calculée sur la base du volume de soude versé.

\section{Mesure de l'activité de la lipase de mésocarpe du palmier à huile}

\section{Mesure sur extrait protéique}

Extraits utilisés pour le dosage de l'activité lipase. Pour le dosage de l'activité lipase, il est utilisé des extraits protéiques de mésocarpe de fruits frais ou lyophilisés. L'extrait peut être également délipidé ou purifié.

Le fruit peut être stérilisé avec de l'hypochlorite de sodium $(0,5 \%)$ et rincé avec de l'eau distillée pour s'assurer de l'élimination de toutes lipases exogènes. Après avoir enlevé le péricarpe, des fragments de mésocarpe sont prélevés sur les fruits frais. Dans le cas où le mésocarpe frais est broyé (pâte de mésocarpe) et directement utilisé, il s'agit d'un extrait brut trop riche en lipides, ce qui pose un problème de stabilité de l'extrait et de l'activité lipase. Ngando Ebongue et al. (2006) ont montré que l'activité lipase est stabilisée après lyophilisation du mésocarpe. En effet, les fragments de mésocarpe sont immédiatement introduits dans de l'azote liquide puis lyophilisés à une température de $-80^{\circ} \mathrm{C}$ pendant environ trois jours. Ils sont alors broyés et ce broyat peut servir d'extrait pour doser l'activité lipase. L'extrait lyophilisé peut également subir une délipidation selon des protocoles précis. Un extrait stable une semaine à $4{ }^{\circ} \mathrm{C}$ et plusieurs semaines à $-20^{\circ} \mathrm{C}$ a été obtenu après dilapidation partielle de la pulpe lyophilisée et suspension dans l'hexane, ce qui a permis d'éliminer le maximum (plus de $80 \%$ ) de lipides susceptibles de perturber les calculs d'activités spécifiques tout en conservant l'activité de l'enzyme (Ngando Ebongue et al., 2006 ; Ngando Ebongue, 2009).

Ces extraits ne répondent pas aux exigences d'un véritable extrait enzymatique utilisé pour la caractérisation biochimique qui doit être totalement purifié.

Méthode directe de test de l'activité lipase. On utilise soit des méthodes basées sur la mesure radioactive sur des substrats radio-marqués, soit une méthode titrimétrique notamment le $\mathrm{pH}$-stat, la technique de référence pour le dosage de l'activité et la spécificité des lipases. Les méthodes de dosage mentionnées dans la littérature (dosages à l'aide de substrats radio-marqués) ne permettent pas une détermination des valeurs absolues d'activités spécifiques de la lipase, compte tenu de la présence de quantités importantes de lipides endogènes dans les différents extraits enzymatiques utilisés lors des dosages (Ngando Ebongue, 2009). Pour pallier cet état de chose, Ngando Ebongue et al. (2006) ont mis au point une méthode fiable et utilisable en routine basée sur un solvant (hexane) et des extraits de mésocarpe lyophilisés permettant d'obtenir des fractions avec une activité lipase plus stable, dans laquelle l'activité lipase 
est mesurée à l'aide du pH-stat en dosant les acides gras libérés avec de l'hydroxyde de sodium $(\mathrm{NaOH})$.

Mesures indirectes: mesures in situ

La mesure de l'activité lipase peut être déterminée de façon indirecte à travers la formation d'AGL dans le fruit occasionnée par l'activation de la lipase. Il s'agit d'une mesure in situ consistant à induire, par différentes méthodes, l'activité de l'enzyme dans les fruits frais puis extraire l'huile issue du mésocarpe et procéder au dosage titrimétrique de l'acidité dans l'huile. En effet, une induction par exposition des fruits à $5{ }^{\circ} \mathrm{C}$ a été utilisée par Sambanthamurthi et al. (1995) qui ont extrait de l'huile à différents intervalles de temps et procédé au dosage titrimétrique de l'acidité de l'huile. De même, après récolte, les fruits frais peuvent être conservés au réfrigérateur à $5{ }^{\circ} \mathrm{C}$ pendant $24 \mathrm{~h}$ pour une activation maximale de l'enzyme et cette dernière est inactivée en stérilisant les fruits pendant $1 \mathrm{~h}$ dans un autoclave (Cadena et al., 2013). Il est également possible de broyer le mésocarpe dans un mortier, extraire de l'huile à différents intervalles de temps et réaliser le dosage titrimétrique de l'acidité de l'huile (Desassis, 1957).

La méthode de congélation et décongélation du mésocarpe a été utilisée par Ngando Ebongue et al. (2008) qui ont également extrait de l'huile à différents intervalles de temps et procédé au dosage titrimétrique de son acidité.

\subsection{Utilité de la mesure de l'acidité pour le sélectionneur}

Étant donné que le caractère « acidité » est plus facile à caractériser, avec des mesures assez fiables et robustes, et compte tenu de la forte corrélation positive qui existe entre l'acidité de l'huile extraite d'un fruit soumis à un stress (broyage, exposition au froid, congélation) et l'activité de la lipase endogène (Ngando Ebongue et al., 2008 ; Morcillo et al., 2013), l'acidité de l'huile peut être considérée comme le caractère le plus accessible et le plus utile pour le sélectionneur pour apprécier la diversité génétique de la teneur en AGL dans le mésocarpe ou l'activité de la lipase endogène du mésocarpe chez le palmier à huile. Il reste néanmoins important de noter que la corrélation entre activité enzymatique et acidité n'est probablement pas linéaire à cause du manque de stabilité de l'enzyme et le fait que le substrat disponible pour l'enzyme est probablement limitant dans le cas des tests in situ.

\section{LES LIPASES ENDOGÈNES CHEZ LE PALMIER À HUILE}

Chez le palmier à huile, il a été identifié deux lipases endogènes majeures, une lipase de germination (Oo,
1981) et une lipase du mésocarpe du fruit (Desassis, 1957 ; Abigor et al., 1985 ; Sambanthamurthi et al., 1991; Sambanthamurthi et al., 1995; Ngando Ebongue et al., 2006). La lipase de germination est située au niveau de l'embryon de la graine et est impliquée dans la mobilisation des réserves nutritives (les triglycérides essentiellement) indispensables à la croissance post-germinative. Quant à la lipase du mésocarpe, son existence est longtemps restée controversée car certains auteurs ont soutenu qu'une lipase endogène du mésocarpe était à la base de la dégradation des TAG, alors que pour d'autres auteurs cette dégradation des TAG n'était due qu'à l'activité microbienne. Étant donné qu'il est aujourd'hui connu que la lipase endogène joue le rôle principal dans l'acidification, l'attention de la recherche s'est plutôt dirigée vers la lipase endogène du mésocarpe.

\subsection{Mise en évidence de la lipase du mésocarpe}

Selon Desassis (1957), l'acidification de l'huile dans les fruits est due à la présence d'une lipase endogène du mésocarpe. Il rapporte que 15 min suffisent pour dégrader $40 \%$ des triglycérides de l'huile du mésocarpe préalablement broyé. D'autres auteurs indiquent cependant une cause microbienne et soutiennent soit l'absence de toute lipase endogène du mésocarpe, soit un rôle majeur de lipases microbiennes (Oo, 1981 ; Tombs \& Stubbs, 1982 ; Hiol et al., 2000 ; Abbas et al., 2002). Coursey (1963) conclut que l'action des lipases microbiennes serait insignifiante comparativement à celle de la lipase endogène. Abigor et al. (1985) ont procédé à la purification et à la caractérisation partielle d'une lipase endogène active du mésocarpe. Ils concluent que cette lipase est associée à la membrane des oléosomes et qu'elle se retrouve au contact des lipides lors de dommages causés aux fruits. Cette localisation histologique a été confirmée par les travaux de Mohankumar et al. (1990). L'activité de la lipase démarre vers 16-20 semaines après anthèse, ce qui coïncide avec le début de la synthèse des lipides (Mohankumar et al., 1990 ; Henderson \& Osborne, 1991 ; Sambanthamurthi et al., 1991 ; Sambanthamurthi et al., 1995 ; Corley \& Tinker, 2016). Il n'existe aucune corrélation entre l'activité de la lipase et la teneur en huile du mésocarpe, et les composants du rendement en huile ne sont pas affectés chez les palmiers à faible acidité de l'huile (Morcillo et al., 2013), donnant ainsi peu d'importance à une hypothétique implication de la lipase dans la synthèse de l'huile. Partant du fait que l'huile dans le mésocarpe ne participe pas au métabolisme de la plante, mais sert à attirer les animaux disséminateurs, Morcillo et al. (2013) ont spéculé sur la possibilité que la lipase $\mathrm{du}$ mésocarpe pourrait permettre d'augmenter la palatabilité des graisses, liée en partie aux récepteurs 
aux acides gras et non aux TAG, amenant ainsi à ce que les fruits attirent davantage les animaux.

Les travaux de Ngando Ebongue et al. (2006) ont définitivement confirmé l'existence de cette lipase et son implication dans l'acidification de l'huile de palme. Ils ont aussi mis au point une méthode fiable permettant d'obtenir une activité stable de l'enzyme afin de la caractériser. Celle-ci leur a permis de caractériser l'activité lipase de génotypes de palmier à huile et établir une corrélation positive entre l'activité lipase et l'acidité.

Pour lever l'équivoque quant à la contribution de la lipase endogène et celle des micro-organismes dans l'acidité de l'huile, une étude consistant à comparer un lot de régimes traités au fongicide après récolte à des témoins non traités a montré que la part de l'acidification due à la lipase endogène était prépondérante sur l'acidification microbienne (Morcillo et al., 2013). Ceci vient confirmer les résultats de plusieurs autres études dans ce sens (Sambanthamurthi et al., 1995 ; Ngando Ebongue, 2009 ; Ngando-Ebongue et al., 2013).

\subsection{Caractérisation de la lipase du mésocarpe}

Les trois groupes (Henderson \& Osborne, 1991; Sambanthamurthi et al., 1995 ; Ngando Ebongue et al., 2006) qui ont caractérisé l'activité lipase en utilisant un triglycéride à longue chaine comme substrat ont tous travaillé sur des extraits protéiques bruts, délipidés ou non. Les résultats de ces trois groupes vont dans le même sens.

Ngando Ebongue et al. (2006) ont montré qu'une température de $35^{\circ} \mathrm{C}$ et un $\mathrm{pH}$ basique de 9 étaient des conditions optimales de réaction. Dans ces conditions optimales, l'activité spécifique a été de 33 mol d'AGL par minute et par mg de protéine, et le mésocarpe contient environ 190 unités enzymatiques de lipase par $\mathrm{g}$ de poids frais (Ngando Ebongue et al., 2006). Ces données suggèrent que la lipase pourrait représenter environ $1 \%$ des protéines totales du mésocarpe. Par ailleurs, Ngando Ebongue et al. (2006) ont testé l'effet de certaines composantes du milieu réactionnel (éthylène diamine tétra-acétate : $\mathrm{EDTA}, \mathrm{CaCl}_{2}, \mathrm{MgCl}_{2}$ ) sur l'activité lipase en modifiant par ajout, suppression ou combinaison de certaines composantes, le milieu réactionnel standard $\left(\mathrm{NaCl} 150 \mathrm{mM}, \mathrm{CaCl}_{2} 10 \mathrm{mM}\right.$, Tris- $\mathrm{HCl} 2 \mathrm{mM}$ et $10 \mathrm{ml}$ d'une émulsion d'huile d'olive de $10 \%[\mathrm{v} / \mathrm{v}]$ dans une solution de gomme arabique $[1 \%, \mathrm{w} / \mathrm{v}])$. Ils ont montré qu'avec l'un ou l'autre des substrats utilisés (huile d'olive, tributyrine), l'EDTA inhibe totalement l'activité lipase, mais le $\mathrm{CaCl}_{2}$ a un effet de restauration de ladite activité, d'où l'importance du calcium dans le dosage de l'activité lipase du mésocarpe du palmier à huile selon les auteurs. La lipase peut être inhibée par certaines substances ou des composés chimiques tels que le diéthyl-p-nitrophénylphosphate (E600), les dérivés des acides boroniques et surtout la tétrahydrolipstatine (THL) qui agit sur le site actif de la sérine (Hadvary et al., 1988 ; Ngando Ebongue et al., 2006).

Morcillo et al. (2013) ont procédé par une approche de protéomique fonctionnelle qu'ils ont mise au point en utilisant la THL radioactive pour marquer les lipases dans un extrait brut de protéine. En effet, la THL peut s'unir de manière covalente au residu de la sérine catalytique de la lipase. Par autoradiographie, ils ont détecté une seule bande de protéine de $55 \mathrm{KDa}$. La séquence de la protéine marquée a pu être établie après analyse protéomique et est identique à la séquence codée par FLL1 (Nurniwalis et al., 2007). Elle contient la séquence consensus «ILVTGHSLGG » des lipases (motif PROSITE PS0120) et inclut le résidu de sérine active $\left(\mathrm{S}_{308}\right)$ formant le motif $\mathrm{GxSxG}$ (Nurniwalis et al., 2015) contenu dans toutes les enzymes des hydrolases alpha/beta. Ensemble avec les résidus d'acide aspartique $\left(\mathrm{D}_{368}\right)$ et d'histidine $\left(\mathrm{H}_{464}\right)$, ils forment la triade catalytique putative Ser-Asp-His indispensable aux lipases pour leur activité d'hydrolyse des acides gras de lipides complexes (Li et al., 2012). Morcillo et al. (2013) ont alors montré, en utilisant des anticorps spécifiques, que la lipase était absente dans les fruits des arbres dont le mésocarpe contient une très faible activité lipase (voir plus bas), ce qui suggère très fortement qu'il s'agit bien de la lipase responsable en grande partie de l'acidité de l'huile de palme.

\section{AMÉLIORATION GÉNÉTIQUE}

La plus importante cause d'acidification étant la dégradation due à la lipase endogène du mésocarpe, la mise au point d'un matériel végétal ne produisant pas ou très peu d'acidité est devenue une des priorités des sélectionneurs. Plusieurs travaux se sont intéressés à ces aspects du sujet.

\subsection{Diversité génétique de l'acidité de l'huile de palme : identification de sources à faible acidité}

Le genre Elaeis comporte deux espèces: l'espèce africaine (E. guineensis) et l'américaine (E. oleifera). Plusieurs auteurs ont caractérisé l'acidité après avoir soumis des échantillons de fruits de ce matériel végétal à des conditions optimales pour l'activation de la lipase et l'obtention du degré maximal d'acidité. Dans le cas d'obtention d'une teneur maximale d'AGL (acidité maximale), les individus ayant moins de $20 \%$ d'AGL dans le mésocarpe du fruit peuvent être considérés comme faiblement acides (Domonhedo, 2010 ; Morcillo et al., 2013). Sambanthamurthi et al. (2000b) ont caractérisé la teneur en AGL d'une centaine de génotypes de la collection du MPOB (Malaysian Palm 
Oil Board), essentiellement des individus issus de trois origines d'E. guineensis (Cameroun, Angola et Tanzanie) et de trois origines d'E. oleifera (Surinam, Brésil et Honduras). Pour ce faire, l'activité lipase a été induite en exposant les fruits à $5^{\circ} \mathrm{C}$ jusqu'au lendemain, comme décrit dans Sambanthamurthi et al. (1991), conditions dans lesquelles la teneur en AGL atteint un niveau maximal. Ils ont montré que cette acidité varie entre 3,2 et $66,8 \%$ chez les palmiers E. guineensis et de 2,9 à $6,2 \%$ chez les E. oleifera. L'espèce E.oleifera présente une faible acidité et E. guineensis une plus grande diversité de teneur en AGL. Après avoir activé la lipase du mésocarpe en procédant à la blessure des fruits soumis à des cycles de congélation et décongélation, Ngando Ebongue et al. (2008) ont caractérisé l'activité lipase et l'acidité de 39 génotypes de palmiers à huile de matériels en cours d'amélioration de l'IRAD (Institut de Recherche Agricoles pour le Développement du Cameroun), issus de plusieurs origines d'E. guineensis et d'une origine de l'espèce E. oleifera. Il ressort également de leurs travaux que les individus $E$. guineensis présentent une large gamme de teneurs en AGL (5-55 \%) incluant des individus à faible acidité, tandis que les individus de E. oleifera ont une faible acidité (8-10\%). De même, Wong et al. (2015) ont caractérisé la teneur en AGL de 148 palmiers issus de différentes origines africaines en activant la lipase selon la méthode décrite par Sambanthamurthi et al. (1991) et ont identifié des individus présentant une acidité maximale comprise entre 0,26 et $65 \%$. Quant à Cadena et al. (2013), ils ont rapporté aussi une très faible teneur en AGL $(<0,6 \%)$ chez E. oleifera. Il existe donc dans différents fonds génétiques d'E. guineensis des individus avec des teneurs en AGL significativement différentes indiquant que ce caractère est génotype dépendant. Ceci constitue ainsi une piste pour la sélection de génotypes à faible activité lipase chez $E$. guineensis. Le tableau 1 présente la variabilité de la teneur en AGL dans quelques fonds génétiques où des arbres à faible acidité d'huile ont été identifiés.

S'agissant des hybrides interspécifiques (E.guineensis X E.oleifera), Cadena et al. (2013) indiquent à partir d'individus issus de trois croisements hybrides que la teneur en AGL varie également selon les génotypes $(0,8-58 \%)$ et se rapproche plus de la gamme de valeurs obtenues chez les E. guineensis, ce qui suggère une dominance partielle de l'E. guineensis sur l'E. oleifera pour ce caractère.

Ces caractérisations peuvent être étendues à beaucoup d'autres fonds génétiques du palmier à huile, pour mieux rechercher des sources de faible acidité et identifier le statut des géniteurs fondateurs. Il est rapporté dans la littérature que l'activité lipase est faible chez l'espèce E. oleifera, mais il n'existe pas d'étude portant sur un ensemble de matériels assez diversifiés et représentatifs.

Une étude associant l'utilisation de marqueurs d'ADN est aussi nécessaire pour renforcer l'analyse de la variabilité phénotypique et permettre de localiser les zones du génome qui contrôlent ce caractère pour faire de la sélection assistée par marqueurs. Ceci pourra aussi

Tableau 1. Variabilité de la teneur en acides gras libres (AGL) dans des origines et fonds génétiques différents de palmier à huile - Variability of free fatty acids (FFA) of different origins and genetic background of oil palm.

\begin{tabular}{|c|c|c|}
\hline Origines ou fonds génétiques & Valeur d'acidité (\% AGL) & Sources \\
\hline Elaeis guineensis : Cameroun, Angola et Tanzanie & $3,2-66,8$ & Sambanthamurthi et al., $2000 \mathrm{~b}$ \\
\hline Elaeis oleifera : Surinam, Brésil, Honduras & $2,9-6,2$ & Sambanthamurthi et al., $2000 \mathrm{~b}$ \\
\hline Elaeis oleifera : Monteria & $8-10$ & Ngando Ebongue et al., 2008 \\
\hline $\begin{array}{l}\text { Elaeis guineensis : La Mé, Yangambi, Deli x La Mé, } \\
\text { Deli x Yangambi }\end{array}$ & $5-55$ & Ngando Ebongue et al., 2008 \\
\hline Elaeis oleifera & $<0,6$ & Cadena et al., 2013 \\
\hline Hybride interspécifique Coari x La Mé & $0,8-58$ & Cadena et al., 2013 \\
\hline $\begin{array}{l}\text { Elaeis guineensis : Angola, Deli x La Mé, Deli x Avros, } \\
\text { Deli x Nigeria, Deli x Ghana, Deli x Yangambi }\end{array}$ & $20-65$ & Cadena et al., 2013 \\
\hline Elaeis guineensis : Deli (DA115D) & $0-80$ & $\begin{array}{l}\text { Domonhédo, } 2010 \text {; } \\
\text { Morcillo et al., } 2013\end{array}$ \\
\hline $\begin{array}{l}\text { Elaeis guineensis: Guinée, Nigéria, Sénégal, Angola, } \\
\text { Cameroun, Tanzanie, Zä̈re (Congo) }\end{array}$ & $0,26-65$ & Wong et al., 2015 \\
\hline
\end{tabular}

Les valeurs présentées sont obtenues dans des conditions d'activation de la lipase et d'obtention d'une acidité maximale. Dans ces conditions, les individus ayant jusqu'à $20 \%$ d'AGL peuvent être considérés comme faiblement acides - Values presented here are potential maximal acidity obtained after lipase activation. In this condition, individuals with until $20 \%$ of FFA can be considered as of low acidity (Domonhédo, 2010 ; Morcillo et al., 2013). 
consolider les fonds porteurs de la « faible acidité » ou révéler d'autres fonds et individus à faible acidité.

\subsection{Identification de facteurs génétiques responsables de l'acidité}

À partir d'une collection d'EST ( « Expressed Sequence Tags ») de mésocarpe de 17 semaines après anthèse, Nurniwalis et al. $(2007,2008)$ ont identifié un gène candidat (FLL1) codant pour une protéine de la famille des lipases de classe 3 , avec un niveau d'expression très élevé au cours du développement du mésocarpe et en particulier durant la synthèse de l'huile. FLL1 contenait un cadre ouvert de lecture (séquence de triplets de nucléotides délimitée par un codon initiateur et un codon stop et pouvant être traduite) de $1452 \mathrm{pb}$, pour lequel a été prédit une séquence polypeptidique de 483 acides aminés. Tranbarger et al. (2011) et Morcillo et al. (2013) ont aussi isolé du mésocarpe un transcrit de lipases de classe 3, nommé EgLIP1. Nurniwalis et al. (2015) ont par la suite montré que FLL1 et EgLIP1 correspondaient au même gène. Morcillo et al. (2013) ont aussi montré que la hausse de l'activité lipase et des quantités de transcrit et de protéine de EgLIP1 lors de l'accumulation des lipides s'observait chez des individus à huile fortement acide mais pas chez des individus à faible acidité. Ceci confirme la corrélation entre l'activité de cette lipase et l'acidité de l'huile mise en évidence par Ngando Ebongue et al. (2008).

Par ailleurs, l'identification de ce gène est cohérente avec les résultats des études ayant porté sur le mode de transmission de l'acidité de l'huile, qui ont montré que ce caractère était essentiellement sous le contrôle d'un gène (Domonhédo, 2010 ; Morcillo et al., 2013 ; Likeng-Li-Ngue et al., 2016). Selon Domonhédo (2010), la faible acidité serait liée à un allèle récessif de ce gène chez E. guineensis. Cette hypothèse a été confirmée par plusieurs auteurs (Morcillo et al., 2013 ; Likeng-Li-Ngue et al., 2016). Un marqueur microsatellite (mEgCIR_LIP03) localisé dans le gène EgLIP1, sur le chromosome 3 selon la séquence du génome entier de Singh et al. (2013), qui co-ségrège avec l'acidité de l'huile dans la pulpe, a été identifié, confortant l'idée qu'EgLIP1 est le gène contrôlant ce caractère, ou qu'il est étroitement lié à d'autres facteurs le contrôlant (Morcillo et al., 2013). Ces travaux ont porté sur quelques familles issues d'un seul arbre de la population Deli. Ce marqueur du gène EgLIP1 est une ressource utile pour la sélection et il est important de confirmer son utilité sur une diversité plus large et d'affiner la recherche d'éventuels autres gènes impliqués.

Le déterminisme génétique est probablement plus complexe car la variation observée au sein d'un même fonds génétique, et notamment la présence de sousclasses avec des acidités significativement différentes dans du matériel végétal à faible acidité, suggère l'implication de plus d'un gène, c'est-à-dire un gène majeur et un ou des gène $(\mathrm{s})$ mineur(s) (Domonhédo, 2010). Wong et al. (2015) indiquent qu'au moins deux gènes codant pour des lipases putatives s'expriment dans le mésocarpe du palmier à huile et suggèrent qu'en dehors de EgLIP1, d'autre(s) gènes(s) détermineraient la qualité de l'huile.

\subsection{Vers un développement de variétés à faible acidité}

Le réseau actuel de sélectionneurs et producteurs de semences de palmier à huile permet de fournir directement du matériel amélioré aussi bien aux palmeraies industrielles qu'aux petites exploitations. La mise à disposition de matériel à faible acidité peut être facilitée par ce réseau et permettra une augmentation du rendement et de la qualité de l'huile, et une gestion plus flexible des récoltes, du traitement et de l'extraction de l'huile. En effet, Morcillo et al. (2013) estiment que les pertes en termes d'acides gras libres pourraient être réduites, même avec un retard à la récolte de 10 jours, ce qui fournirait un rendement en huile additionnel de 2-3\% expliqué par une maturation optimale, associée à une réduction des fréquences de récoltes. Prenant en compte ces impacts, les mêmes auteurs évaluent à 1 milliard de dollars par an le gain économique que cela pourrait représenter. Par ailleurs, l'utilisation d'un matériel à faible acidité permettra, notamment aux petits producteurs/transformateurs, de réduire la fréquence de récolte et de gérer à leur rythme avec plus de souplesse les opérations de traitement des régimes et d'extraction de l'huile. À notre connaissance, les premiers palmiers à huile commerciaux à faible acidité ont été produits dans les champs semenciers de l'Institut National des Recherches Agricoles du Bénin (INRAB) à Pobè et distribués à partir de 2015 (PalmElit, 2016).

Par ailleurs, il est important d'approfondir les travaux sur l'identification des gènes ou autres facteurs génétiques afin de disposer d'informations plus précises sur le déterminisme génétique et moléculaire de ce caractère. La mise en évidence d'autres gènes faciliterait ainsi une sélection pour réduire encore l'acidité de l'huile de palme. Les résultats précédemment obtenus ont essentiellement tenu compte de l'espèce E. guineensis. Il faudra étendre les études à l'espèce E. oleifera et conclure sur la transmission de ce caractère chez le genre Elaeis et confirmer les ressources génomiques utilisables pour l'amélioration génétique sur l'ensemble de ces espèces. Bien que chez l'espèce E. oleifera, l'huile issue du mésocarpe du fruit est très peu acide, il est difficile d'envisager un programme de réduction de l'acidité par rétrocroisements successifs ([E.guineensis X E. oleifera $] \times$ E.guineensis), car il 
nécessiterait plusieurs décennies d'investissement. Il est donc nécessaire de mieux cerner le mécanisme de transmission chez cette espèce également, puis valider éventuellement les outils génomiques en vue d'envisager une sélection assistée par marqueur à l'échelle du genre, pour amener de manière plus efficace à des sorties variétales à faible acidité.

\section{CONCLUSIONS}

L'acidification de l'huile affecte la qualité et la stabilité de l'huile de palme. Elle est principalement due à la lipase endogène du mésocarpe, mais aussi à l'action de micro-organismes. Elle est donc affectée par des facteurs génétiques intrinsèques à la plante, mais aussi par les méthodes de gestion des récoltes, de traitement des régimes, d'extraction et de conservation de l'huile. Un travail d'amélioration génétique est possible notamment chez E. guineensis au regard de la grande variabilité observée pour ce caractère au sein de cette espèce. Ceci améliorera la qualité de l'huile tout en augmentant le rendement et en facilitant la gestion des opérations de récolte et de post-récolte, en particulier pour les petits producteurs/transformateurs. Plusieurs travaux ont été réalisés et ouvrent des pistes de recherche pour mieux préciser la transmission de ce caractère chez le genre Elaeis et disposer de différentes ressources génomiques capables de faire une sélection en routine plus efficace et plus rapide pour la faible acidité. Plusieurs travaux ont été entrepris et ont abouti récemment à la commercialisation par l'INRAB et PalmElit des premiers palmiers avec une huile faiblement acide.

\section{Bibliographie}

Abbas H., Hiol A., Deyris V. \& Comeau L., 2002. Isolation and characterization of an extracellular lipase from Mucor sp. strain isolated from palm fruit. Enzym. Microbiol. Technol., 31, 968-975.

Abigor D.R., Opoku R.A., Opute F.I. \& Osagie A.U., 1985. Partial purification and some properties of the lipase present in oil palm (Elaeis guineensis) mesocarp. J. Sci. Food Agric., 36, 599-606.

Beisson F., Tiss A., Rivière C. \& Verger R., 2000. Methods for lipase detection and assay : a critical. Eur. J. Lipid Sci.Technol., 1, 133-153.

Berger K.G., 1983. Problems in palm oil handling and storage. In: Proceedings of the Regional Workshop, Palm Oil Mill Technology and Effluent Treatment, 1718 August 1982, Palm Oil Research Institute, Kuala Lumpur, Malaysia, 112-118.

Cadena T., Prada F., Perea A. \& Romero H.M., 2013. Lipase activity, mesocarp oil content, and iodine value in oil palm fruits of Elaeis guineensis, Elaeis oleifera, and the interspecific hybrid $\mathrm{O} \times \mathrm{G}($ E. oleifera $\times$ E. guineensis $)$. J. Sci. Food Agric., 93(3), 674-680.

Carlsson A.S. et al., 2011. Replacing fossil oil with fresh oil - with what and for what? Eur. J. Lipid Sci. Technol., 113, 812-831.

Chong C.L., 2000. Storage, handling and transportation of palm oil and palm oil products. In: Basiron Y., Jalani B.S. \& Chan K.W., eds. Advances in oil palm research. Vol. 2. Kuala Lumpur: Malaysian Palm Oil Board, 806-844.

CNUCED, 2016. Huile de palme - Un profil de produit de base, http://unctad.org/fr/PublicationsLibrary/ INFOCOMM_cp08_PalmOil_fr.pdf, (06.08.2017).

Codex Alimentarius, 2015. Norme pour les huiles végétales portant un nom spécifique. CODEX STAN 210-1999. Adoptée en 1999. Amendement: 2005, 2011, 2013, 2015. Révision : 2001, 2003, 2009. Rome : FAO/OMS.

Corley R.H.V. \& Tinker P.B., 2016. The oil palm. $5^{\text {th }}$ ed. Oxford, UK: John Wiley \& Sons, Ltd.

Coursey A., 1963. The deterioration of palm oil during storage. J. West Africa Sci. Assoc., 7, 101-114.

de Graaf J., 1976. Composition, quality and end uses of palm oil. In: Corley R.H.V., Hardon J.J. \& Wood B.J., eds. Oil palm research. Amsterdam, The Netherlands: Elsevier, 493-503.

Demol J. et al., 2002. Le palmier à huile (Elaeis guineensis Jacq.). In: Demol J. et al., eds. Amélioration des plantes, application aux principales espèces cultivées en régions tropicales. Gembloux, Belgique : Les Presses agronomiques de Gembloux, 1-23.

Desassis A., 1957. L'acidification de l'huile de palme. Oléagineux, 12, 525-534.

Domonhédo H., 2010. Déterminisme génétique et sélection de palmier à huile (Elaeis guineensis Jacq.) à faible acidité en République du Bénin. Mémoire de DEA: Université d'Abomey-Calavi (Bénin).

Durand-Gasselin T. et al., 2010. Sélection du palmier à huile pour une huile de palme durable et responsabilité sociale. Ol. Corps Gras Lipides, 17, 385-392.

Durand-Gasselin T. et al., 2011. Breeding for sustainable palm oil. In: Proceedings of the International Seminar on Breeding for Sustainability in Oil Palm, 18 November 2011, Kuala Lumpur, Malaysia, 178-193.

Eastmond P.J., 2006. SUGAR-DEPENDENT1 encodes a patatin domain triacylglycerol lipase that initiates storage oil breakdown in germinating Arabidopsis seeds. Plant Cell, 18(3), 665-675.

Egloff M.-P. et al., 1995. Enzymes lipolytiques et lipolyse. Ol. Corps Gras Lipides, 2(52).

Fickers P., Destain J. \& Thonart P., 2008. Les lipases sont des hydrolases atypiques: principales caractéristiques et applications. Biotechnol. Agron. Soc. Environ., 12(2), 119-130.

Gibon V. et al., 2009. Future prospects for palm oil refining and modifications. Ol. Corps Gras Lipides, 16(4), 193-200. 
Gibon V., De Greyt W. \& Kellens M., 2007. Palm oil refining. Eur. J. Lipid Sci. Technol., 109(4), 315-335.

Hadvary P., Lengsfeld H. \& Wolfer H., 1988. Inhibition of pancreatic tetrahydrolipstatin in vitro by the covalent inhibitor tetrahydrolipstatin. Biochem. J., 256, 357361.

Haryati T. \& Buana L., 2003. Determination of free fatty acid content of palm oil through its $\mathrm{pH}$. In: Proceedings of the International Palm Oil Conference on Progress, Prospects and Challenges towards the $21^{\text {st }}$ century, 1428 August 2003, Kuala Lumpur, Malaysia.

Henderson J. \& Osborne D., 1991. Lipase activity in ripening and mature fruit of the oil palm. Stability in vivo and in vitro. Phytochemistry, 30, 1073-1078.

Hills M.J. \& Mukherjee K., 1990. Triacylglycerol lipase from rape (Brassica napus) suitable for biotechnological purposes. Appl. Biochem. Biotechnol., 26, 1-10.

Hiol A. et al., 2000. Purification and characterization of an extracellular lipase from a thermophilic Rhizopus oryzae strain isolated from palm fruit. Enzym. Microb. Technol., 26, 421-430.

Huang A.H.C., 1993. Lipases. In: Moore T.S., ed. Lipid metabolism in plants. Boca Raton, FL, USA: CRC Press, 473-503.

Kelly A.A. \& Feussner I., 2016. Oil is on the agenda: lipid turnover in higher plants. Biochim. Biophys. Acta, 1861(9), 1253-1268.

Li W. et al., 2012. Analysis of Arabidopsis genes encoding putative class III lipases. J. Plant Biochem. Biol., 21, 261-267.

Likeng-Li-Ngue B.C. et al., 2016. Genetic determinism of oil acidity among some DELI oil palm (Elaeis guineensis Jacq.) progenies. Afr. J. Biotechnol., 15(34), 1841-1845.

Loncin M. \& Jacobsberg B., 1963. Studies on Congo palm oil. J. Am. Oil Chem. Soc., 40, 18-45.

Mohankumar C., Arumughan C. \& Kaleysa Raj R., 1990. Histological localization of oil palm fruit lipase. J. Am. Oil Chem. Soc., 67, 665-669.

Morcillo F. et al., 2013. Improving palm oil quality through identification and mapping of the lipase gene causing oil deterioration. Nat. Commun., 4, ID 2160.

Murphy D.J., 2009. Oil palm: future prospects for yield and quality improvements. Lipid Technol., 21, 257-260.

Ngando Ebongue G.F., 2009. Étude de la lipase endogène du mésocarpe du fruit du palmier à huile (Elaeis guineensis Jacq.) : application à la sélection de lignées à faible acidité de l'huile. Thèse de doctorat : Université Victor Segalen Bordeaux 2 (France).

Ngando Ebongue G.F. et al., 2006. Assaying lipase activity from oil palm fruit (Elaeis guineensis Jacq.) mesocarp. Plant Physiol. Biochem., 44(10), 611-617.

Ngando Ebongue G.F. et al., 2008. Identification of oil palm breeding lines producing oils with low acid values. Eur. J. Lipid Sci. Technol., 110(6), 505-509.

Ngando Ebongue G.F., Mpondo Mpondo E.A., Dikotto Ekwe E.L.\& Paul K., 2011. Assessment of the quality of crude palm oil from smallholders in Cameroon. J. Stored Prod. Postharvest Res., 2(3), 52-58.

Ngando Ebongue G.F., Mpondo Mpondo E.A., Dikotto Ekwe E.L. \& Koona P., 2013. Some quality parameters of crude palm oil from major markets of Douala, Cameroon. Afr. J. Food Sci., 7(12), 473-478.

Nurniwalis A.W., Siti Nor Akmar A., Chan P.L. \& Manaf M.A., 2007. Isolation of a cDNA encoding a lipase class 3 family protein from oil palm (Elaeis guineensis, Jacq.). In: Proceedings of the PIPOC 2007 International Palm Oil Congress, Agriculture, Biotechnology \& Sustainability, 26-30 August 2007, Kuala Lumpur, Malaysia, 1011-1020.

Nurniwalis A.W. et al., 2008. Gene discovery via expressed sequence tags from the oil palm (Elaeis guineensis, Jacq.) mesocarp. J. Oil Palm Res., 2, 87-96.

Nurniwalis A.W. et al., 2015. Genomic structure and characterization of a lipase class 3 gene and promoter from oil palm. Biol. Plant., 59(2), 227-236.

Ohimain I.E., Daokoru-Olukole C., Izah C.S. \& Alaka E.E., 2012. Assessment of the quality of crude palm oil produced by smallholder processors in Rivers State, Nigeria. Niger. J. Agric. Food Environ., 8(2), 28-34.

Oo K.C., 1981. The absence of lipase activity in mesocarp of the oil palm fruit. Oléagineux, 36, 613-616.

Orji M.U. \& Mbata T.I., 2008. Effect of extraction methods on the quality and spoilage of Nigerian palm oil. Afr. J. Biochem. Res., 2(9), 192-196.

PalmElit, 2016. « Low Lipase », un progrès pour toute la filière du palmier à huile. Newsletter, 3 .

Parakh R.L. \& Pathak M.P., 2000. The palm oil refining and palm products manufacturing - an experience. In: Pushparajah E., ed. Proceedings of the International planters conference plantation tree crops in the new millenium: the way ahead, 17-20 Mai 2000, Kuala Lumpur, Malaysia, 835-842.

Rival A. \& Levang P., 2014. Palms of controversies: oil palm and development challenges. Bogor, Indonesia: CIFOR.

RSPO, 2017. Smallholders, http://www.rspo.org/ smallholders, (04/08/2017).

Sambanthamurthi R. et al., 1991. Chilling-induced lipid hydrolysis in the oil palm (Elaeis guineensis) mesocarp. J. Exp. Bot., 42, 1199-1205.

Sambanthamurthi R., Oo K. \& Parman S., 1995. Factors affecting lipase activity in Elaeis guineensis mesocarp. Plant Physiol. Biochem., 33, 353-359.

Sambanthamurthi R., Sundram K. \& Tan Y., 2000a. Chemistry and biochemistry of palm oil. Prog. Lipid Res., 39, 507-558.

Sambanthamurthi R., Rajanaidu N. \& Parman S.H., 2000b. Screening for lipase activity in the oil palm. Biochem. Soc. Trans., 28(6), 769-770.

Singh R. et al., 2013. Oil palm genome sequence reveals divergence of interfertile species in Old and New worlds. Nature, 500(7462), 335-339. 
Soumanou M., 1986. Influence du mode de préparation sur la qualité et la stabilité de l'huile de palme. Mémoire d'ingénieur : Université d'Abomey-Calavi (Bénin).

Tombs M. \& Stubbs J.M., 1982. The absence of lipase from oil palm mesocarp. J. Sci. Food Agric., 33, 892-897.

Tranbarger T.J. et al., 2011. Regulatory mechanisms underlying oil palm fruit mesocarp maturation, ripening, and functional specialization in lipid and carotenoid metabolism. Plant Physiol., 156, 564-584.

USDA, 2017. Oilseeds: world market and trade. Foreign Agricultural Service, Circular Series March 2017, http:// apps.fas.usda.gov/psdonline/circulars/production.pdf, (16/03/2017).

Vermeulen S. \& Goad N., 2006. Towards better practice in smallholder palm oil production. Natural Resource Issues Series No. 5. London: International Institute for Environment and Development.

Wong Y.T. et al., 2015. Screening of wild oil palm (Elaeis guineensis) germplasm for lipase activity. J. Agric. Sci., 154(7), 1241-1252.

(61 réf.) 